Ähnliche Fragen haben $\mathrm{Schwiersch}{ }^{5}$ sowie $\mathrm{Be}-$ li a nkin und Dilator s ky ${ }^{6}$ diskutiert.

Um eine Verschiedenheit im Wassergehalt kann es sich hier aber nicht handeln. Denn Häutchen verschiedener Dicken wurden verschieden lang nach ihrer Herstellung - 2 Stdn. bis 2 Monate - untersucht und zeigten keine Änderung ihrer Lichtbrechungseigenschaften. Wenn es sich um $\gamma-\mathrm{Al}_{2} \mathrm{O}_{3}$ mit verschiedenem Wassergehalt handeln würde, so müßte man aus den Brechungsquotienten den Wassergehalt bestimmen können. Nimmt man den theoretischen Wert 1,7330 für $\gamma-\mathrm{Al}_{2} \mathrm{O}_{3}$ nach $\mathrm{B}$ e lin a nk in und Dilatorsky6, und nimmt man ferner an, daß

\begin{tabular}{|c|c|}
\hline $\begin{array}{c}\text { Erzeugungsspannung } \\
\text { in Volt }\end{array}$ & $\begin{array}{c}\text { Brechungs- } \\
\text { quotient }\end{array}$ \\
\hline 90 & 1,567 \\
100 & 1,573 \\
110 & 1,576 \\
120 & 1,582 \\
130 & 1,587 \\
140 & 1,590 \\
150 & 1,592 \\
160 & 1,596 \\
170 & 1,601 \\
180 & 1,603 \\
190 & 1,611 \\
200 & 1,614 \\
\hline
\end{tabular}

Tab. 1. Lichtbrechung verschiedener $\mathrm{Al}_{2} \mathrm{O}_{3}-\mathrm{Häutchen}$ $\left(t=18^{\circ} \mathrm{C}\right.$, Na-Dampflampe).

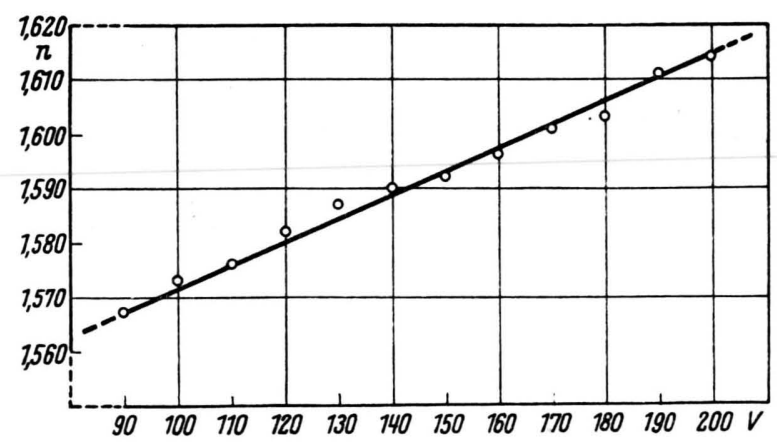

Abb. 1. Brechungsquotient als Funktion der Spannung.

die Abhängigkeit des Brechungsquotienten vom Wassergehalt linear ist, so ergäbe sich für die bei $200 \mathrm{~V}$ hergestellten Häutchen ein Wassergehalt von $30 \%$, für die bei $90 \mathrm{~V}$ hergestellten ein solcher von $42 \%$. Beide Werte sind unwahrscheinlich. Ebensowenig scheint bei den ähnlichen Herstellungsbedingungen ein Unterschied im Wassergehalt von $12 \%$ plausibel.

Ein anderer Grund für die Änderung der Brechungsquotienten der untersuchten Häutchen kann die Dicke oder aber die Erzeugungsspannung und damit die Packungsdichte sein. Es wurde versucht, bei der Herstellung der Häutchen durch stufenweise Steigerung der

6 D. Beliankin u. N. Dilat orsky, Zbl. Mineral., Geol., Paläont., Abt. A 229 [1932].
Spannung bis zum Endwert eine Klärung dieser Frage herbeizuführen. Es wurden verschiedene Spannungsstufen gewählt und der Brechungsquotient der erhaltenen Schicht bestimmt.

Erzeugungs- Brechungs- Erzeugungs- Brechungsspannung quotient spannung quotient

$\begin{array}{rrrr}200 \mathrm{~V} & 1,614 & 150 \mathrm{~V} & 1,592 \\ 50+50+50+50 \mathrm{~V} & 1,614 & 50+50+50 \mathrm{~V} & 1,593 \\ 100+60+40 \mathrm{~V} & 1,613 & 100+50 \mathrm{~V} & 1,594\end{array}$

Diese Werte zeigen, daß sich die Lichtbrechung und damit wohl auch die Schichtdicke so einstellt, als ob von Anfang an die höchste Spannung angelegt worden wäre. Eine Entscheidung kann dieses Ergebnis nicht bringen, es kann aber einen Hinweis auf die Bildungsweise des $\mathrm{Al}_{2} \mathrm{O}_{3}$ Häutchens auf Al-Folien geben.

Wenn hier der Ansicht der Vorzug gegeben wird, daß die Dicke für die Änderung der Lichtbrechung maßgebend ist, so deshalb, weil damit die Diskrepanzen in der Lichtbrechung von Objekten, die an der Auflösungsgrenze des Mikroskops liegen, besonders der Tonminerale, erklärbar wären.

Für die Herstellung der $\mathrm{Al}_{2} \mathrm{O}_{3}$-Häutchen bin ich Herrn Dipl.-Phys. S tro h m a i e r und Herrn cand. phys. S a i le r zu Dank verpflichtet.

\section{Über die Registrierung von Konzentrationsgradienten nach dem Minimalstrahlverfahren}

Von O. Armbruster, W. Kossel und $\mathrm{K}$. $\mathrm{S}$ troh ma i e r

Physikalisches Institut der Universität Tübingen und Max-Planck-Institut für Biochemie, Tübingen

(Z. Naturforschg. 6 a, 510-511 [1951]; eingeg. am 13. Aug. 1951)

Die Wanderung von Kolloiden im elektrischen Feld oder ihre Sedimentation in der Ultrazentrifuge wird durch direkte Beobachtung mit Hilfe der Töplerschen Schlierenmethode verfolgt. Zur Bestimmung der Konzentration und der Wanderungs- bzw. Sedimentationsgeschwindigkeit werden genau auswertbare Kurven, in denen $\mathrm{d} n / \mathrm{d} x$ gegen $x$ aufgetragen ist ( $x=$ vom Kolloid zurückgelegte Strecke) nach der Skalenmethode von $\mathrm{L} \mathrm{a} \mathrm{m} \mathrm{m}{ }^{1}$ erhalten. Da die Auswertung der Aufnahmen bis zum Vorliegen der fertigen Kurven nach dieser Methode sehr viel Zeit beansprucht, wurde von verschiedenen Autoren versucht, durch Modifikation der ursprünglichen Töplerschen Anordnung direkte Aufzeichnung dieser Kurven zu erhalten. Die bekannteste dieser Methoden ist die Registrierung nach Philpot-Svensson. Dabei wird die bei der Töplerschen Schlierenmethode verwendete Kante schräg gestellt und schließlich durch einen Spalt oder Draht ersetzt. Der Verlauf des Konzentrationsgefälles in der Küvette wird dann als Verlauf einer hell oder dunkel gezeichneten Kurve wiedergegeben. Sie ist von starken Beugungserscheinungen begleitet, die die Genauigkeit der Beobachtung sehr beeinträchtigen können. Abb. 1 zeigt an einem Extremfall eine derartige Kurvenverzerrung, wie

1 O. Lamm, Z. physik. Chem. Abt. A 138, 313 [1928]. 
sie des öfteren zu beobachten ist. Dies wird besonders bei Anwendung des Spaltes recht störend, weshalb sich A n t weiler ${ }^{2}$ veranlaßt sah, ein Verfahren auszuarbeiten, um den wirklichen Kurvenverlauf rekonstruieren zu können.

Hier weist das Minimalstrahlverfahren, das W o l t e r ${ }^{3}$. angegeben hat, einen neuen Weg. Er verwendet zur Strahldefinition an Stelle eines Spaltes eine Phasenkante, die zwar auf beiden Seiten den Lichtstrom frei durchläßt, ihn jedoch auf der einen Seite so verzögert, daß ein Gangunterschied von $\lambda / 2$ zu dem unbehinderten Teil entsteht. Im Beugungsbild einer Phasenkante tritt ein Minimum besonders hervor ${ }^{4}$, das bei dieser Phasenverzögerung um $180^{\circ}$ in die Mitte rückt und dort eine vollständige Auslöschung hervorbringt. Dieses Minimum ist zum Unterschied von dem mit dem Draht entstehenden einfach. Wegen des logarithmischen Verlaufs der Helligkeitsempfindung des Auges und der Möglichkeit, das Minimum auf der photographischen Platte durch Überexposition der Umgebung scharf einzuengen, erscheint es viel schärfer als das ebenfalls durch Beugung mitbestimmte helle Bild eines Spaltes. Die seitlich neben ihm liegenden Beugungsstreifen sind bei einer absorptionslosen Phasenkante, die um $\lambda / 2$ verzögert, symmetrisch und stören viel weniger als bei Draht- oder Spaltbildern. Abb. $1-4^{*}$ zeigen eine Gegenüberstellung von Aufnahmen desselben Konzentrationsgradienten mit Hilfe der besprochenen Methoden. Die Apparatur war auf große Empfindlichkeit justiert, im Falle geringerer Empfindlichkeit geht das durch die Phasenkante erzeugte Bild in das mit Hilfe des Drahtes hergestellte über.

Wie bei jeder Minimumaufzeichnung tritt jedoch die Schwierigkeit auf, daß bei gleichzeitiger Aufnahme zweier Linien - etwa der Konzentrations- und einer Bezugslinie - das Minimum der einen in das Hellfeld der anderen fällt, so daß die Kontraste sich gegenseitig schwächen. Dem wird begegnet, wenn man das Hellfeld begrenzt, indem man die Phasenkante in die Mitte eines verhältnismäßig breiten Spaltes setzt. Es erscheint dann eine scharfe dunkle Linie in einem breiteren hellen Streifen (Abb. 4). Eine Kcntrastminderung ist nun ausgeschlossen, wo ein Minimum nicht geradezu von einer anderen hellen Linie überschnitten wird. Als Phasenkante können verwendet werden freitragende Aluminiumoxydhäutchen 5 oder beliebiges durchsichtiges Material, das auf die erforderliche Dicke gebracht werden kann, wie auf Glas aufgezogene Kollodiumfolien o. ä. Sehr stabil gegen mechanische Beanspruchung ist eine Glasplatte, die zur Hälfte mit Material entsprechender Dicke bedampft ist, wie es bei der Erzeugung von Antireflexschichten angewandt wird.

Die Methode, die nur einen geringfügigen Zusatz zur Apparatur erfordert, wird nun seit Monaten in der laufenden Arbeit an der Elektrophorese-Apparatur des MaxPlanck-Instituts für Biochemie ständig angewandt und hat sich als dem bisherigen Verfahren durchaus überlegen bewährt.

2 H. J: A n tw eil e r, Angew. Chem. 62, 412 [1950].

3 H. Wolter, Ann. Physik 7, 341 [1950].

4 W. K o s s e l u. K. S t r o h m a i e r, Z. Naturforschg. 6 a, 504 [1951].

5 K. S trohma ier, Diplomarbeit Tübingen 1950; Z. Naturforschg. 6a, 508 [1951].

* Abb. 1-4 s. Tafel S. 512 a.

\section{Zur Beseitigung störender Aufladungen nichtleitender Objekte und Leuchtschirme im Elektronenstrahl}

\author{
Von G. Kn o e r z e r \\ Physikalisches Institut der Universität Tübingen \\ (Z. Naturforschg. 6 a, 511-512 [1951]; eingeg. am 13. Aug. 1951)
}

Die störende Wirkung, die die Aufladung eines Leuchtschirms durch den Elektronenstrahl auf die Abbildung ausüben kann, zeigt sich schon beim Schattenwurf einfacher Objekte in sehr charakteristischer Form. So zeigt unsere Abb. 1* in der Richtung des geometrischen Schattens einer einfachen Drahtspirale Aufhellung, sobald diese geerdet ist (a), und verbreiterten Schatten, sobald man sie isoliert (b). Im zweiten Fall ist ohne weiteres deutlich, daß der aufgehängte Draht sich mit dem Vorzeichen der Elektronen aufgeladen hat - das Schattenbild ist daclurch ver-

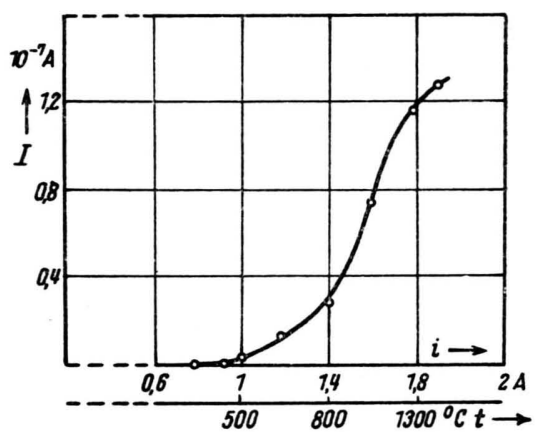

Abb. 3. Abhängigkeit des Emissionsstromes $I$ vom Heizstrom $i$ bei einem Draht von $0,15 \mathrm{~mm}$. Die untere Skala gibt die Temperatur in ${ }^{\circ} \mathrm{C}$.

breitert und die abgedrängten Elektronen umranden es mit einem Maximum von Helligkeit. In Abb.1 a wirkt zunächst überraschend, daß der geerdete Draht als konzentrierende Linse arbeitet (bis auf einige offenbar mit Oxyd belegte Stücke, die sich aufgeladen haben), besonders deutlich in den hübschen Kaustiken, die sich da zeigen, wo der Strahl an einer Windung entlangstreift. Das für die Erscheinung verantwortliche Feld kann hier nur vom Leuchtschirm selbst ausgehen, und dies wird unmittelbar bestätigt, wenn man ihn mit einer für die Elektronen noch durchlässigen Metallhaut bedampft. Das Bild 1 a erscheint, solange diese isoliert bleibt. Beim Erden zeigt sich, wie bekannt, normales Schattenbild. Man konstruiert leicht, wie hier die Konzentration der vom Schirm kommenden Kraftlinien am Draht zu einer Zylinderlinsenwirkung(1 a) führt.

Die gesamten Folgen der negativen Aufladung verschwinden sofort, wenn ein außerhalb des Strahlweges vor dem Schirm angebrachter Wolframdraht zum Glühen gebracht wird - man erhält ein völlig korrektes Schattenbild (Abb. 2).

Diese Wirkung überrascht dadurch, daß eine negative Ladung vom Schirm entfernt wird. Es kann sich also nicht um die Glühelektronen des Drahtes handeln, sondern es

* Abb. $1 \mathrm{a}, 1 \mathrm{~b}$ und 2 siehe Tafel S. $512 \mathrm{~b}$. 